\section{Point: The Imprecise Pursuit of Precision Medicine: Are Biomarkers to Blame?}

Bishal Gyawali, MD

Precision medicine is the concept of tailoring treatment to individual patients based on the presence or absence of certain objectively measurable parameters known as biomarkers. These biological markers can be predictive (ie, tell whether a given drug will work) or prognostic (ie, tell how better or worse the cancer outcomes will be regardless of treatment) or both. Precision medicine (ie, personalized therapy or tailored treatment) has gained enormous popularity because it aims to improve the benefit/risk profile of cancer treatment. Providing the drug only to patients who are likely to benefit and avoiding the drug in those who are not is certainly an attractive concept.

In reality, however, we don't seem to be achieving as much tangible result from this approach as promised. Experts have openly questioned the value of precision medicine-and rightly so, because the only randomized controlled trial (RCT) of precision medicine to date has provided negative results. ${ }^{1,2}$ Why has such an attractive approach failed to improve outcomes? This is a logical question to ask.

Although it may well be that the concept of precision medicine is fallacious, it would be prudent to first look at biomarkers, because if there are errors in measurement of a biomarker or questions about its validity, the whole framework of precision medicine crumbles. Precision medicine is impossible without precision in the measurement and validation of biomarkers.

However, accurate measurement of biomarkers is not easy. There are many potential avenues for errors. The case history of excision repair cross-complement group 1 protein (ERCC1) as a biomarker for platinum therapy in non-small cell lung cancer (NSCLC) illustrates these problems very well. ERCC1 has been touted as a predictive biomarker for platinum therapy in NSCLC for many years, and is now even available commercially, but no evidence supports or refutes this claim.

A recent study mapped all the research activities concerning ERCC1 over the past 12 years. ${ }^{3}$ During this period, 28 studies of ERCC1 as a predictive biomarker for platinum therapy in NSCLC were reported. Of these, only 2 were prospective studies. Putting all the studies over 12 years together using what the authors called the "Accumulating Evidence and Research Organization" (AERO) model helped recognize major flaws in the ERCC1 research: most studies were poorly designed, the studies used different methods to measure ERCC1 levels, the cutoff points used were different across studies, the chemotherapy regimens were not uniform, and there has been little replication or validation of techniques. Thus, despite considerable investment in terms of time, money, human power, and logistics for this research over 12 years, we are unable to derive any conclusions.

In this context, the ERCC1 Trial was an important study; it was the first prospective phase III study assessing both the predictive and prognostic utility of ERCC1 for squamous and nonsquamous NSCLC. ${ }^{4}$ This study found no utility of ERCC1 as a predictive or prognostic marker for platinum therapy in NSCLC. Although at first look the results suggest that this properly conducted phase III RCT settled the question of whether ERCC1 is a predictive marker of benefit from platinum therapy in NSCLC, a problem remains: this study measured ERCC1 levels using 8F1 assay. However, the accuracy of $8 \mathrm{~F} 1$ assay in measuring ERCC1 levels has been questioned. Experiments have shown that ERCC1 is not the principal antigen recognized by the $8 \mathrm{~F} 1$ antibody and that $8 \mathrm{~F} 1$ does not discriminate between ERCC1-positive and

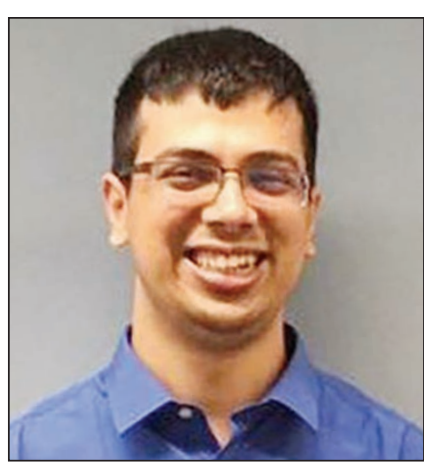

Bishal Gyawali, MD

Bishal Gyawali, MD, is a medical oncologist with experience in cancer care in both low-income and high-income countries. After graduating from medical school in Nepal with 7 gold medals, he is currently in the final year of his postgraduate training and $\mathrm{PhD}$ in medical oncology at Nagoya University Graduate School of Medicine, Japan, as a MEXT scholar. Dr. Gyawali is a medical consultant at the not-for-profit Anticancer Fund, Belgium, and also holds a faculty affiliation at the Institute of Cancer Policy, London, United Kingdom. His original research and opinion articles have been published in top oncology journals. He also provides critical commentary on oncology updates every month at his blogs on the ecancer Web site (http://ecancer.org/). He is an editorial adviser to $B M J$, and a Journal of Global Oncology Editorial Fellow for 2017. His areas of clinical and research interests include evidence-based oncology practice, global oncology, cancer policy, cancer management in resource-limited settings, financial toxicities of cancer treatment, clinical trial methods, and supportive treatments of cancer. Dr. Gyawali tweets at @ oncology_bg. 
ERCC1-deficient nuclei. ${ }^{5}$ Thus, whether ERCC1 measured by other methods would have produced similar results is unknown. Therefore, even after phase III evidence from a well-designed study, the answer to whether ERCC1 can be used as a predictive marker in NSCLC remains elusive.

This problem of validity in measuring biomarkers is relevant to modern immune checkpoint inhibitors. PD-1 inhibitors such as nivolumab and pembrolizumab are arguably some of the most expensive cancer drugs. These drugs are truly useful in some cancers, but only in a subset of patients. For this subset, PD-1 inhibitors can sometimes provide durable responses, but for most patients they do not provide much benefit. In fact, these drugs cause many adverse events, some of which can be serious or even fatal, including the huge burden of financial toxicity. Thus, we need biomarkers that can help determine which patients benefit from this therapy and which only experience toxicities. In the absence of predictive biomarkers, we are potentially exposing all eligible patients to toxicities (including financial).

In this context, PD-L1 levels have been proposed as a putative biomarker. ${ }^{6}$ In fact, for the first-line treatment of NSCLC, pembrolizumab is approved only in patients whose tumor PD-L1 levels are $\geq 50 \%$. However, PD-L1 as a biomarker is unreliable, with significant temporal, spatial, and methodologic heterogeneity. ${ }^{7}$ For the same patient, PD-L1 levels may be different when biopsies are taken from the same tumor at different times, or even from different areas in the same tumor sample. PD-L1 levels also differ depending on which antibody or approach was used for measurement. ${ }^{7}$ This heterogeneity makes the measurement of PD-L1 levels very tricky and clinical decisionmaking problematic. Further complicating the matter, the clinical trials of nivolumab and pembrolizumab have used different methods to measure PD-L1 expression levels.

In conclusion, until we achieve a consensus in the measurement of biomarkers, promoting the idea of precision medicine may be premature. Precision medicine requires precise measurement and validation of biomarkers. Imprecise biomarker measurement can lead to the erroneous conclusion that personalized medicine is ineffective, when the issue could simply be the imprecision in measurement of biomarkers. Until the biomarkers have been proven with analytical and external validity, exercises in biomarker-based precision medicine are likely to be futile.

\section{Author Response to Counterpoint Commentary by Kato et al (page 863)}

Until today, the debate of precision oncology has revolved around whether it improves outcomes in our patients. Based on the available data, the answer is that it does not - at least to the extent that it has been sold. The aim of this commentary was to consider whether imprecision in the measurement and validity of biomarkers is one of the reasons why the promise of precision oncology has not been delivered. However, in another commentary in this issue of JNCCN (page 863) Kato et al propose that precision oncology has already achieved success and that biomarkers are already precise. Where I find the need for caution and further work, Kato et al see the need for celebration. They tell us that biomarkers fully deserve the credit for the stellar success of precision oncology, but in real life, patients who receive precision oncology care continue to die of cancer, and do so having suffered many questionable treatments.

I sincerely believe that both I and the experts with opposing views want our patients to win; it doesn't matter whether the patients' win is achieved through precision oncology or elsewhere. If it comes through precision oncology, it's all the more better. That's precisely why I ask for precision in measuring biomarkers. 
In their defense of precision oncology, Kato et al offer examples of success such as that achieved with the use of imatinib in chronic myeloid leukemia (CML) or epidermal growth factor receptor (EGFR) inhibitors in EGFR-positive lung cancer. However, there is a fundamental flaw in that argument: it fallaciously assumes that when I or other critics question precision oncology, ${ }^{1,2,8}$ we criticize the validated targeted therapies, such as imatinib in CML or trastuzumab in HER2-positive breast cancer. These are examples of remarkable progress in oncology; something we in the oncology field are proud of. My criticism is against the next-generation sequencing (NGS)-based genomic profiling or omics-based testing, and tailoring treatment based on those matches without having evidence to support such approaches. This "modern" definition of precision oncology is where I differ with Kato and others. ${ }^{9}$ In other words, the success examples listed in Table 1 (page 865) of the Kato et al commentary justifies the testing of only those biomarkers and not the rampant genomic-based testing and treatment. Indeed, one could argue that for all the noises of success of precision oncology, the only ones with data are those the authors listed in their table; the rest have not delivered the promise.

Kato et al criticized the SHIVA trial. I agree that SHIVA is not an ideal trial, but until the criticism comes in the form of a better trial, it's the only trial we have. Indeed, the only data the authors can offer in support of their argument regarding the success of NGS-based precision oncology are from a couple of metaanalyses of phase I and II trials, but no randomized studies.

Kato et al presented a nice argument in their commentary that " $80 \%$ of patients in SHIVA received monotherapy with either an mTOR inhibitor or a hormone modulator. Hence, it is reasonable to conclude that these 2 types of single agents given to patients with advanced refractory cancer are not effective. It is not justified to extrapolate from this limited data and conclude that all of precision medicine is a failure." By the same token, the countable few successes achieved with BRAF inhibitors in melanoma, HER2 inhibitors in breast cancer, imatinib in CML or gastrointestinal stromal tumors (GIST), and ALK and EGFR inhibitors in lung cancer cannot be touted as success of the whole of precision oncology as an approach.

The results of the MOSCATO trial have recently been published and paint a further sobering picture for the current state of precision oncology. ${ }^{10}$ The authors report only $7 \%$ of patients who were successfully screened benefited with this approach. Considering a total number of 948 patients who were biopsied and 1,035 patients included, only $2.32 \%$ and $2.12 \%$, respectively, received benefit. This is a very sobering success rate for an approach that has been eulogized so much. And even this marginal "success" rate is from a nonrandomized design.

In conclusion, it is premature to celebrate the success of precision oncology, especially when we lack data from even a single RCT to support this strategy. It is also premature to burn the effigy of precision oncology. Despite the negative data from a single RCT, and because biomarker-based treatments have seen some success in some cancers, precision oncology should be given another chance through conducting an RCT. If precision oncology is as good as we have believed it to be, there should be no hesitation that it can stand the test of an RCT. But if it is not, we would be harming many patients by using an unproven strategy. However, before we perform the RCT, it might be prudent to pause and ponder whether it is the imprecision in biomarkers that is failing us rather than precision oncology as a strategy. 
Gyawali

\section{References}

1. West HJ. No solid evidence, only hollow argument for universal tumor sequencing: show me the data. JAMA Oncol 2016;2:717-718.

2. Prasad V. Perspective: the precision-oncology illusion. Nature 2016;537:S63.

3. Barsanti-Innes B, Hey SP, Kimmelman J. The challenges of validating in precision medicine: the case of excision repair cross-complement group 1 diagnostic testing. Oncologist 2017;22:89-96.

4. Lee SM, Falzon M, Blackhall F, et al. Randomized prospective biomarker trial of ERCC1 for comparing platinum and nonplatinum therapy in advanced non-small-cell lung cancer: ERCC1 Trial (ET). J Clin Oncol 2017;35:402411.

5. Niedernhofer LJ, Bhagwat N,Wood RD. ERCC1 and non-small-cell lung cancer. N Engl J Med 2007;356:25382540.

6. Patel SP, Kurzrock R. PD-L1 expression as a predictive biomarker in cancer immunotherapy. Mol Cancer Ther 2015;14:847-856

7. McLaughlin J, Han G, Schalper KA, et al. Quantitative assessment of the heterogeneity of PD-L1 expression in non-small-cell lung cancer. JAMA Oncol 2016;2:46-54.

8. Tannock IF, Hickman JA. Limits to personalized cancer medicine. N Engl J Med 2016;375:1289-1294.

9. Prasad V, Gale R. What precisely is precision oncology—and will it work? Available at: http://www.ascopost.com/ issues/january-25-2017/what-precisely-is-precision-oncology-and-will-it-work/. Accessed January 25, 2017.

10. Massard C, Michiels S, Ferté $C$, et al. High-throughput genomics and clinical outcome in hard-to-treat advanced cancers: results of the MOSCATO 01 trial. Cancer Discov 2017;7:586-595. 